\title{
Breaking Value Symmetry ${ }^{\star}$
}

\author{
Toby Walsh \\ NICTA and UNSW \\ Sydney, Australia \\ tw@cse.unsw.edu.au
}

\section{Introduction}

One common type of symmetry is when values are symmetric. For example, if we are assigning colours (values) to nodes (variables) in a graph colouring problem then we can uniformly interchange the colours throughout a colouring. For a problem with value symmetries, all symmetric solutions can be eliminated in polynomial time [1,2]. However, as we show here, both static and dynamic methods to deal with symmetry have computational limitations. With static methods, pruning all symmetric values is NP-hard in general. With dynamic methods, we can take exponential time on problems which static methods solve without search.

\section{Background}

A constraint satisfaction problem consists of a set of variables, each with a domain of values, and a set of constraints specifying allowed combinations of values for given subsets of variables. A solution is an assignment of values to variables satisfying the constraints. Variables take one value from a given finite set. Symmetry occurs in many constraint satisfaction problems. A value symmetry is a permutation of the values that preserves solutions. More formally, a value symmetry is a bijective mapping $\sigma$ on the values such that if $X_{1}=d_{1}, \ldots, X_{n}=d_{n}$ is a solution then $X_{1}=\sigma\left(d_{1}\right), \ldots, X_{n}=$ $\sigma\left(d_{n}\right)$ is also. A variable symmetry, on the other hand, is a permutation of the variables that preserves solutions. More formally, a variable symmetry is a bijective mapping $\sigma$ on the indices of variables such that if $X_{1}=d_{1}, \ldots, X_{n}=d_{n}$ is a solution then $X_{\sigma(1)}=d_{1}, \ldots, X_{\sigma(n)}=d_{n}$ is also. Symmetries are problematic as they increase the size of the search space. For instance, if we have $m$ interchangeable values, symmetry increases the size of the search space by a factor of $m$ !.

Many constraint solvers explore the space of partial assignments enforcing some local consistency. We consider four local consistencies for finite domain variables Given a constraint $C$, a support is assignment to each variable of a value in its domain which satisfies $C$. A constraint is generalized arc consistent $(G A C)$ iff for each variable, every value in its domain belongs to a support. A set of constraints is GAC iff each constraint

\footnotetext{
* NICTA is funded by the Australian Government's Department of Communications, Information Technology and the Arts and the Australian Research Council through Backing Australia's Ability and the ICT Centre of Excellence program. Thanks to Chris Jefferson and Jean-Francois Puget for useful comments.
} 
is GAC. On binary constraints, GAC is simply called arc consistency (AC). A set of binary constraints is singleton arc consistent (SAC) iff we can assign every variable with each value in its domain and make the resulting problem arc consistent (AC). Finally, a set of binary constraint is $k$-consistent iff each $k-1$ assignment can be consistently extended to a $k$ th variable, and is strongly $k$-consistent iff it is $j$-consistency for all $j \leq k$. We will compare local consistency properties applied to sets of constraints, $c_{1}$ and $c_{2}$ which are logically equivalent. As in [4], a local consistency property $\Phi$ on $c_{1}$ is as strong as $\Psi$ on $c_{2}$ iff, given any domains, if $\Phi$ holds on $c_{1}$ then $\Psi$ holds on $c_{2} ; \Phi$ on $c_{1}$ is stronger than $\Psi$ on $c_{2}$ iff $\Phi$ on $c_{1}$ is as strong as $\Psi$ on $c_{2}$ but not vice versa; $\Phi$ on $c_{1}$ is equivalent to $\Psi$ on $c_{2}$ iff $\Phi$ on $c_{1}$ is as strong as $\Psi$ on $c_{2}$ and vice versa.

\section{Static methods}

One simple and common mechanism to deal with symmetry is to add constraints which eliminate symmetric solutions [5]. Suppose we have a set $\Sigma$ of value symmetries. Based on [6], we can eliminate all symmetric solutions by posting a global constraint which ensures that the solution is ordered lexicographically before any of its symmetries. More precisely, we post the global constraint $\operatorname{VALSYMBREAK}\left(\Sigma,\left[X_{1}, \ldots, X_{n}\right]\right)$ which ensures $\left[X_{1}, \ldots, X_{n}\right] \leq_{\operatorname{lex}}\left[\sigma\left(X_{1}\right), \ldots, \sigma\left(X_{n}\right)\right]$ for all $\sigma \in \Sigma$ where $X_{1}$ to $X_{n}$ is a fixed ordering on the variables. Unfortunately, pruning all values from such a symmetry breaking constraint is NP-hard.

Theorem 1 Deciding if $\operatorname{ValSymBreak}\left(\Sigma,\left[X_{1}, \ldots, X_{n}\right]\right)$ is GAC is NP-complete, even when $|\Sigma|$ is linearly bounded.

Proof: Membership in NP follows by giving a support for every possible assignment. To prove it is NP-hard, we give a reduction from a 3-SAT problem in $N$ Boolean variables and $M$ clauses. We construct a CSP with $N+M+1$ variables over $4 N+2$ possible values. The first $4 N$ values partition into $2 N$ interchangeable pairs. The values $4 i-3$ and $4 i-2$ are interchangeable, as are $4 i-1$ and $4 i$ for $1 \leq i \leq N$. The values $4 i-3$ and $4 i-2$ represent $x_{i}$ being true, whilst the values $4 i-1$ and $4 i$ represent $x_{i}$ being false. The final two values, $4 N+1$ and $4 N+2$ are not interchangeable. The first $N$ CSP variables represent a "truth assignment". We have $X_{i} \in\{4 i-3,4 i-2,4 i-1,4 i\}$ for $1 \leq i \leq N$. The next $M$ CSP variables ensure at least one literal in each clause is true. For example, if the $i$ th clause is $x_{j} \vee \neg x_{k} \vee x_{l}$, then the domain of $X_{N+i}$ is $\{4 j-3,4 j-2,4 k-1,4 k, 4 l-3,4 l-2\}$. The final variable $X_{N+M+1}$ is a "switch" and has the domain $\{4 N+1,4 N+2\}$. Note that all variables have symmetric domains.

We have two sets of constraints. First, we have the constraints $\operatorname{odd}\left(X_{N+M+1}\right) \rightarrow$ $\operatorname{odd}\left(X_{i}\right)$ for $1 \leq i \leq N$ and $\operatorname{odd}\left(X_{N+M+1}\right) \rightarrow \operatorname{even}\left(X_{N+j}\right)$ for $1 \leq j \leq M$. Second, we have the constraints odd $\left(X_{N+M+1}\right) \rightarrow P H P(N, N+1)$ and even $\left(X_{N+M+1}\right) \rightarrow$ $\operatorname{PHP}(N, N)$ where $\operatorname{PHP}(i, j)$ is a pigeonhole constraint which holds iff the variables $X_{1}$ to $X_{i}$ take $j$ distinct values. Note that $\operatorname{PHP}(N, N+1)$ is unsatisfiable and that $\operatorname{PHP}(N, N)$ is satisfiable. Thus, the constructed CSP is unsatisfiable if $X_{N+M+1}=$ $4 N+1$ and satisfiable if $X_{N+M+1}=4 N+2$. Note that if we take any solution of the CSP and permute any of the interchangeable values, we still have a solution. Thus, if 
$\Sigma$ is the set of symmetries induced by these interchangeable values, it is permissible to add VALSYMBREAK $\left(\Sigma,\left[X_{1}, \ldots, X_{n}\right]\right)$ to this CSP.

Suppose our branching heuristic sets the switch variable $X_{N+M+1}$ to $4 N+1$. Enforcing AC on the binary constraints prunes the domains of $X_{i}$ to $\{4 i-3,4 i-1\}$ for $1 \leq i \leq N$. Similarly, the domain of $X_{N+i}$ is reduced to $\{4 j-2,4 k, 4 l-2\}$. Consider now finding a support for VALSYMBREAK given this particular subproblem. $X_{N+i}$ can only take the value $4 j-2$ if $X_{j}$ had previously been assigned $4 j-3$. In other words, $X_{N+i}$ can only take the value $4 j-2$ if $x_{j}$ is set to true in the "truth assignment". Similarly, $X_{N+i}$ can only take the value $4 k$ if $X_{k}$ had previously been assigned $4 k-1$. In other words, $X_{N+i}$ can only take the value $4 k$ if $x_{k}$ is set to false in the "truth assignment". Finally, $X_{N+i}$ can only take the value $4 l-2$ if $X_{j}$ had previously been assigned $4 l-3$. In other words, $X_{N+i}$ can only take the value $4 l-2$ if $x_{l}$ is set to true in the "truth assignment". Thus, at least one of the literals in the $i$ th clause must have been set to true in the "truth assignment". Hence, there is a support for VALSYMBREAK iff the original 3-SAT problem is satisfiable. By Theorem 3, $|\Sigma|$ can be linearly bound.

This is a somewhat surprising result. Whilst it is polynomial to eliminate all symmetric solutions either statically [2] or dynamically [1], it is NP-hard to lookahead and prune all symmetric values. Equivalently, whilst we can avoid visiting symmetric leaves of the search tree in polynomial time, avoiding symmetric subtrees is NP-hard.

\section{Dynamic methods}

An alternative to static methods which add constraints to eliminate symmetric solutions are dynamic methods which modify the search procedure to ignore symmetric branches. For example, with value symmetries, the GE-tree method can dynamically eliminate all symmetric leaves in a backtracking search procedure in $O\left(n^{4} \log (n)\right)$ time [1]. However, as we show now, such dynamic methods may not prune all symmetric subtrees which static methods can do. Suppose we are at a particular node in the search tree explored by the GE-tree method. Consider the current and all past variables seen so far. The GE-tree method can be seen as performing forward checking on a static symmetry breaking constraint over this set of variables. This prunes symmetric assignments from the domain of the next branching variable. Unlike static methods, the GE-tree method does not prune deeper variables. By comparison, static symmetry breaking constraints can prune deeper variables, resulting in interactions with the problem constraints and additional domain prunings. For this reason, static symmetry breaking methods can solve certain problems exponentially quicker than dynamic methods.

Theorem 2 There exists a model of the pigeonhole problem with $n$ variables and $n+$ 1 interchangeable values such that, given any variable and value ordering, the GEtree method explores $O\left(2^{n}\right)$ branches, but which static symmetry breaking methods can solve in just $O\left(n^{2}\right)$ time.

Proof: The $n+1$ constraints in the CSP are $\bigvee_{i=1}^{n} X_{i}=j$ for $1 \leq j \leq n+1$, and the domains are $X_{i} \in\{1, \ldots, n+1\}$ for $1 \leq i \leq n$. The problem is unsatisfiable by a simple pigeonhole argument. Any of the static methods for breaking value symmetry 
presented later in this paper will prune $n+1$ from every domain in $O\left(n^{2}\right)$ time. Enforcing GAC on the constraint $\bigvee_{i=1}^{n} X_{i}=n+1$ then proves unsatisfiability. On the other hand, the GE-tree method irrespective of the variable and value ordering, will only terminate each branch when $n-1$ variables have been assigned (and the last variable is forced). A simple calculation shows that the size of the GE-tree more than doubles as we increase $n$ by 1 . Hence we will visit $O\left(2^{n}\right)$ branches before declaring the problem is unsatisfiable.

This theoretical result supports the experimental results in [2] showing that static methods for breaking value symmetry can outperform dynamic methods. Given the intractability of pruning all symmetric values in general, we focus in the rest of the paper on a common and useful type of value symmetry where symmetry breaking methods have been proposed that take polynomial time: we will suppose that values are ordered into partitions, and values within each partition are uniformly interchangeable.

\section{Generator symmetries}

One way to propagate VALSYMBREAK is to decompose it into individual lexicographical ordering constraints, $\left[X_{1}, \ldots, X_{n}\right] \leq_{\operatorname{lex}}\left[\sigma\left(X_{1}\right), \ldots, \sigma\left(X_{n}\right)\right]$ and use one of the propagators proposed in [7] or [8]. Even if we ignore the fact that such a decomposition may hinder propagation (see Theorem 2 in [8]), we have to cope with $\Sigma$, the set of symmetries being exponentially large in general. For instance, if we have $m$ interchangeable values, then $\Sigma$ contains $m$ ! symmetries. To deal with large number of symmetries, Aloul et al. suggest breaking only those symmetries corresponding to generators of the group [9]. Consider the generators which interchange adjacent values within each partition. If the $m$ values partition into $k$ classes of interchangeable values, there are just $m-k$ such generators. Breaking just these symmetries eliminates all symmetric solutions.

Theorem 3 If $\Sigma$ is a set of symmetries induced by interchangeable values, and $\Sigma_{g}$ is the set of generators corresponding to interchanging adjacent values then posting $\operatorname{VALSYMBREAK}\left(\Sigma_{g},\left[X_{1}, \ldots, X_{n}\right]\right)$ eliminates all symmetric solutions.

Proof: Assume $\operatorname{ValSymBreak}\left(\Sigma_{g},\left[X_{1}, \ldots, X_{n}\right]\right)$. Consider any two interchangeable values, $j$ and $k$ where $j<k$, Let $\sigma_{j} \in \Sigma_{g}$ be the symmetry which swaps just $j$ with $j+1$. To ensure $\left[X_{1}, \ldots, X_{n}\right] \leq_{\operatorname{lex}}\left[\sigma_{j}\left(X_{1}\right), \ldots, \sigma_{j}\left(X_{n}\right)\right], j$ must occur before $j+1$ in $X_{1}$ to $X_{n}$. By transitivity, $j$ therefore occurs before $k$. Thus, for the symmetry $\sigma^{\prime}$ which swaps just $j$ with $k,\left[X_{1}, \ldots, X_{n}\right] \leq_{\operatorname{lex}}\left[\sigma^{\prime}\left(X_{1}\right), \ldots, \sigma^{\prime}\left(X_{n}\right)\right]$. Consider now any symmetry $\sigma \in \Sigma$. The proof proceeds by contradiction. Suppose $\left[X_{1}, \ldots, X_{n}\right]>_{\operatorname{lex}}\left[\sigma\left(X_{1}\right), \ldots, \sigma\left(X_{n}\right)\right]$. Then there exists some $j$ with $X_{j}>\sigma\left(X_{j}\right)$ and $X_{i}=\sigma\left(X_{i}\right)$ for all $i<j$. Consider the symmetry $\sigma^{\prime}$ which swaps just $X_{j}$ with $\sigma\left(X_{j}\right)$. As argued before, $\left[X_{1}, \ldots, X_{n}\right] \leq_{\operatorname{lex}}\left[\sigma^{\prime}\left(X_{1}\right), \ldots, \sigma^{\prime}\left(X_{n}\right)\right]$. But this contradicts $\left[X_{1}, \ldots, X_{n}\right]>_{\text {lex }}\left[\sigma\left(X_{1}\right), \ldots, \sigma\left(X_{n}\right)\right]$ as $\sigma$ and $\sigma^{\prime}$ act identically on the first $j$ variables in $X_{1}$ to $X_{n}$. Hence, $\left[X_{1}, \ldots, X_{n}\right] \leq_{\text {lex }}\left[\sigma\left(X_{1}\right), \ldots, \sigma\left(X_{n}\right)\right]$.

Not surprisingly, reducing the number of symmetry breaking constraints to linear comes at a cost. We may not prune all symmetric values. 
Theorem 4 If $\Sigma$ is a set of symmetries induced by interchangeable values, and $\Sigma_{g}$ is the set of generators corresponding to interchanging adjacent values then GAC on $\operatorname{VaLSYmBReaK}\left(\Sigma,\left[X_{1}, \ldots, X_{n}\right]\right)$ is stronger than $G A C$ on $\left[X_{1}, \ldots, X_{n}\right] \leq_{\text {lex }}$ $\left[\sigma\left(X_{1}\right), \ldots, \sigma\left(X_{n}\right)\right]$ for all $\sigma \in \Sigma_{g}$.

Proof: Clearly it is at least as strong. To show it is stronger, suppose all values are interchangeable with each other. Consider $X_{1}=1, X_{2} \in\{1,2\}, X_{3} \in\{1,3\}, X_{4} \in$ $\{1,4\}$ and $X_{5}=5$. Then enforcing $\operatorname{GAC}$ on $\operatorname{VaLSYMBBREA}\left(\Sigma,\left[X_{1}, \ldots, X_{5}\right]\right)$ prunes 1 from $X_{2}, X_{3}$ and $X_{4}$. However, $\left[X_{1}, \ldots, X_{5}\right] \leq_{\text {lex }}\left[\sigma\left(X_{1}\right), \ldots, \sigma\left(X_{5}\right)\right]$ is GAC for all $\sigma \in \Sigma_{g}$.

Finally, it is not hard to see that there are other sets of generators for the symmetries induced by interchangeable values which do not necessarily eliminate all symmetric solutions (e.g. with the generators which interchange the value 1 with any $i$, we do not eliminate either the assignment $X_{1}=1, X_{2}=2$ or the symmetric assignment $X_{1}=1$, $X_{2}=3$ ).

\section{Puget's decomposition}

With value symmetries, a second method that eliminates all symmetric solutions is a decomposition due to [2]. Consider a surjection problem (where each value is used at least once) with interchangeable values. We can channel into dual variables, $Z_{j}$ which record the first index using the value $j$ by posting the binary constraints: $X_{i}=j \rightarrow Z_{j} \leq i$ and $Z_{j}=i \rightarrow X_{i}=j$ for all $1 \leq i \leq n, 1 \leq j \leq m$. We can then eliminate all symmetric solutions by insisting that interchangeable values first occur in some given order. That is, we place strict ordering constraints on the $Z_{k}$ within each class of interchangeable values. Puget notes that any problem can be made into a surjection by introducing $m$ additional new variables, $X_{n+1}$ to $X_{n+m}$ where $X_{n+i}=i$. These variables ensure that each value is used at least once. In fact, we don't need additional variables. It is enough to ensure that each $Z_{j}$ has a dummy value, which means that $j$ is not assigned, and to order (dummy) values appropriately. Unfortunately, Puget's decomposition into binary constraints hinders propagation.

Theorem 5 If $\Sigma$ is a set of symmetries induced by interchangeable values, then GAC on $\operatorname{VaLSYMBReak}\left(\Sigma,\left[X_{1}, \ldots, X_{n}\right]\right)$ is stronger than AC on Puget's decomposition into binary constraints.

Proof: It is clearly at least as strong. To show it is stronger, suppose all values are interchangeable with each other. Consider $X_{1}=1, X_{2} \in\{1,2\}, X_{3} \in\{1,3\}, X_{4} \in$ $\{3,4\}, X_{5}=2, X_{6}=3, X_{7}=4, Z_{1}=1, Z_{2} \in\{2,5\}, Z_{3} \in\{3,4,6\}$, and $Z_{4} \in$ $\{4,7\}$. Then all Puget's symmetry breaking constraints are AC. However, enforcing $\operatorname{GAC}$ on $\operatorname{VaLSYMBREAK}\left(\Sigma,\left[X_{1}, \ldots, X_{5}\right]\right)$ will prune 1 from $X_{2}$.

If all values are interchangeable with each other, we only need to enforce a slightly stronger level of local consistency to prune all symmetric values. More precisely, enforcing singleton arc consistency on Puget's binary decomposition will prune all symmetric values. 
Theorem 6 If all values are interchangeable and $\Sigma$ is the set of symmetries induced by this then GAC on $\operatorname{VaLSYMBReAK}\left(\Sigma,\left[X_{1}, \ldots, X_{n}\right]\right)$ is equivalent to SAC on Puget's decomposition into binary constraints.

Proof: Suppose Puget's encoding is AC. We will show that there is at least one support for VALSYMBREAK. We assign $Z_{1}$ to $Z_{m}$ in turn, giving each the smallest remaining value in their domain, and enforcing $\mathrm{AC}$ on the encoding after each assignment. This will construct a support without the need for backtracking. At each choice point, we ensure that a new value is used as soon as possible, thus giving us the most freedom to use values in the future. Suppose now that Puget's encoding is SAC. Then, by the definition of SAC, we can assign any variable with any value in its domains and be sure that the problem can be made $\mathrm{AC}$ without a domain wipeout. But if the problem can be made AC, it has support. Thus every value in every domain has support. Hence enforcing SAC on Puget's decomposition ensures that VALSYMBREAK is GAC.

We might wonder if singleton arc-consistency is enough for arbitrary value symmetries. That is, does enforcing SAC on Puget's encoding prune all symmetric values? We can prove that no fixed level of local consistency is sufficient. Given the intractability of pruning all symmetric values in general, this result is not surprising.

Theorem 7 For any given $k$, there exists a value symmetry and domains for which Puget's encoding is strongly $k$-consistent but is not $k+1$-consistent.

Proof: We construct a CSP problem with $2 k+1$ variables over $2(k+1)$ possible values. The $2(k+1)$ values partition into $k+1$ pairs which are interchangeable. More precisely, the values $i$ and $k+1+i$ are interchangeable for $1 \leq i \leq k+1$. The first $k$ variables of the CSP have $k+1$ values between them (hence, one value is not taken). More precisely, $X_{i} \in\{i, i+1\}$ for $1 \leq i \leq k$. The remaining $k+1$ variables then take the other $k+1$ values. More precisely, $X_{k+i}=k+1+i$ for $1 \leq i \leq k+1$. The values 1 to $k+1$ need to be used by the first $k$ variables, $X_{1}$ to $X_{k}$ so that the last $k+1$ variables, $X_{k+1}$ to $X_{2(k+1)}$ can use the values $k+2$ to $2(k+1)$. But this is impossible by a pigeonhole argument. Puget's encoding of this is strongly $k$-consistent. since any assignment of $k-1$ or less variables can be extended to an additional variable. On the other hand, enforcing $k+1$-consistency will discover that the CSP has no solution.

Finally, we compare this method with the previous method based on breaking the symmetries corresponding to the generators which interchange adjacent values.

Theorem 8 If $\Sigma$ is a set of symmetries induced by interchangeable values, and $\Sigma_{g}$ is the set of generators interchanging adjacent values then AC on Puget's decomposition for $\Sigma$ is stronger than $G A C$ on $\left[X_{1}, \ldots, X_{n}\right] \leq_{\operatorname{lex}}\left[\sigma\left(X_{1}\right), \ldots, \sigma\left(X_{n}\right)\right]$ for all $\sigma \in \Sigma_{g}$.

Proof: Suppose Puget's decomposition is AC. Consider the symmetry $\sigma$ which interchanges $j$ with $j+1$. Consider any variable and any value in its domain. We show how to construct a support for this assignment. We assign every other variable with $j$ if it is in its domain, otherwise any value other than $j+1$ and failing this, $j+1$. Suppose this is not a support for $\left[X_{1}, \ldots, X_{n}\right] \leq_{\operatorname{lex}}\left[\sigma\left(X_{1}\right), \ldots, \sigma\left(X_{n}\right)\right]$. This means that in the sequence from $X_{1}$ to $X_{n}$, we had to use the value $j+1$ before the value $j$. However, as Puget's decomposition is $\mathrm{AC}$, there is a value in the domain of $Z_{j}$ smaller than $Z_{j+1}$. 
This contradicts $j+1$ having to be used before $j$. Hence, this must be a support. Thus $\left[X_{1}, \ldots, X_{n}\right] \leq_{\text {lex }}\left[\sigma\left(X_{1}\right), \ldots, \sigma\left(X_{n}\right)\right]$ is GAC for all $\sigma \in \Sigma_{g}$. To show that AC on Puget's decomposition is stronger consider again the example used in the proof of Theorem 4. The lexicographical ordering constraint for each generator $\sigma \in \Sigma_{g}$ is GAC without any domain pruning. However, enforcing AC on Puget's decomposition prunes 1 from $X_{2}, X_{3}$ and $X_{4}$.

\section{Value precedence}

A third method to break symmetry due to interchangeable values uses the global precedence constraint [3]. Precedence $\left(\left[X_{1}, \ldots, X_{n}\right]\right)$ holds iff $\min \left\{i \mid X_{i}=j \vee i=\right.$ $n+1\}<\min \left\{i \mid X_{i}=k \vee i=n+2\right\}$ for all $j<k$. That is, the first time we use $j$ is before the first time we use $k$ for all $j<k$. Posting such a constraint eliminates all symmetric solutions due to interchangeable values. In [10], a GAC propagator for such a precedence constraint is given which takes $O(n m)$ time. It is not hard to show that $\operatorname{Precedence}\left(\left[X_{1}, \ldots, X_{n}\right]\right)$ is equivalent to $\operatorname{VaLS} \operatorname{SimBReaK}\left(\Sigma,\left[X_{1}, \ldots, X_{n}\right]\right)$ where $\Sigma$ is the set of symmetries induced by interchangeable values. Hence, enforcing GAC on such a precedence constraint prunes all symmetric values in polynomial time. Precedence constraints can also be defined when values partition into several interchangeable classes; we just insist that values within each class first occur in a fixed order. In [10], a propagator for such a precedence constraint is proposed which takes $O\left(n \prod_{i} m_{i}\right)$ time where $m_{i}$ is the size of the $i$ th class of interchangeable values. This is only polynomial if we can bound the number of classes of interchangeable values. This complexity is now not so surprising. We have shown that pruning all symmetric values is NP-hard when the number of classes of interchangeable values is unbounded.

\section{Related work}

Puget proved that symmetric solutions can be eliminated by the addition of suitable constraints [5]. Crawford et al. presented the first general method for constructing variable symmetry breaking constraints [6]. Petrie and Smith adapted this method to value symmetries by posting a suitable lexicographical ordering constraint for each value symmetry [13]. Puget and Walsh independently proposed propagators for such symmetry breaking constraints $[7,8]$. To deal with the exponential number of such constraints, Puget proposed a global propagator which does forward checking in polynomial time [7]. To eliminate symmetric solutions due to interchangeable values, Law and Lee formally defined value precedence and proposed a specialized propagator for a pair of interchangeable values [3]. Walsh extended this to a propagator for any number of interchangeable values [10]. An alternative way to break value symmetry statically is to convert it into a variable symmetry by channelling into a dual viewpoint and using lexicographical ordering constraints on this dual view [14,12]. A number of dynamic methods have been proposed to deal with value symmetry. Van Hentenryck et al. gave a labelling schema for eliminating all symmetric solutions due to interchangeable values [15]. Inspired by this method, Roney-Dougal et al. gave a polynomial method to construct a GE-tree, a search tree without value symmetry [1]. Finally, Sellmann and van 
Hentenryck gave a $O\left(n d^{3.5}+n^{2} d^{2}\right)$ dominance detection algorithm for eliminating all symmetric solutions when both variables and values are interchangeable [16].

\section{Conclusion}

Value symmetries can be broken either statically (by adding constraints to prune symmetric solutions) or dynamically (by modifying the search procedure to avoid symmetric branches). We have shown that both approaches have computational limitations. With static methods, we can eliminate all symmetric solutions in polynomial time but pruning all symmetric values is NP-hard in general (or equivalently, we can avoid visiting symmetric leaves of the search tree in polynomial time but avoiding symmetric subtrees is NP-hard). With dynamic methods, we typically only perform forward checking and can take exponential time on problems which static methods solve without search.

\section{References}

1. Roney-Dougal, C., Gent, I., Kelsey, T., Linton, S.: Tractable symmetry breaking using restricted search trees. In: Proc. of ECAI-2004, (2004)

2. Puget, J.F.: Breaking all value symmetries in surjection problems. In: Proc. of 11th Int. Conf. on Principles and Practice of Constraint Programming (CP2005), (2005)

3. Law, Y., Lee, J.: Global constraints for integer and set value precedence. In: Proc. of 10th Int. Conf. on Principles and Practice of Constraint Programming (CP2004), (2004)

4. Debruyne, R., Bessière, C.: Some practicable filtering techniques for the constraint satisfaction problem. In: Proc. of the 15th IJCAI, (1997)

5. Puget, J.F.: On the satisfiability of symmetrical constrained satisfaction problems. In: Proc. of ISMIS'93, (1993)

6. Crawford, J., Luks, G., Ginsberg, M., Roy, A.: Symmetry breaking predicates for search problems. In: Proc. of the 5th Int. Conf. on Knowledge Representation and Reasoning (KR '96), (1996)

7. Puget, J.F.: An efficient way of breaking value symmetries. In: Proc. of the 21 st National Conf. on AI, AAAI, (2006)

8. Walsh, T.: General symmetry breaking constraints. In: 12th Int. Conf. on Principles and Practices of Constraint Programming (CP-2006), (2006)

9. Aloul, F., Ramani, A., Markov, I., Sakallah, K.: Solving difficult SAT instances in the presence of symmetries. In: Proc. of the Design Automation Conf, (2002)

10. Walsh, T.: Symmetry breaking using value precedence. In: Proc. of the 17th ECAI, (2006)

11. Bessiere, C., Hebrard, E., Hnich, B., Walsh, T.: The complexity of global constraints. In: Proc. of the 19th National Conf. on AI, AAAI, (2004)

12. Law, Y., Lee, J.: Symmetry Breaking Constraints for Value Symmetries in Constraint Satisfaction. Constraints, 11 (2-3), 221-267 (2006)

13. Petrie, K.E., Smith, B.M.: Symmetry Breaking in Graceful Graphs. Technical Report APES56a-2003, APES Research Group (2003)

14. Flener, P., Frisch, A., Hnich, B., Kiziltan, Z., Miguel, I., Pearson, J., Walsh, T.: Breaking row and column symmetry in matrix models. In: 8th Int. Conf. on Principles and Practices of Constraint Programming (CP-2002), (2002)

15. Hentenryck, P.V., Agren, M., Flener, P., Pearson, J.: Tractable symmetry breaking for CSPs with interchangeable values. In: Proc. of the 18th IJCAI, (2003)

16. Sellmann, M., Hentenryck, P.V.: Structural symmetry breaking. In: Proc. of 19th IJCAI, (2005) 